\title{
Video Authentication and Copyright Protection using Unique Watermark Generation Technique and Singular Value Decomposition
}

\author{
Chhaya S. Gosavi \\ Research Scholar \\ DYPIET, Pimpri, Pune 18 \\ Maharashtra, India
}

\author{
Suresh N. Mali, PhD \\ Principal \\ SITS, Narhe, Pune-41 \\ Maharashtra, India
}

\begin{abstract}
Digital watermark is perceptually invisible information embedded in the video. This embedding is done by an encoder using a secret key. The watermark can carry information about owner or recipient of the video or the video itself or some additional information like video caption, date etc. The watermarked video may undergo possible changes by unauthorized users or attackers. Unintentional and malicious attacks are aimed to disable watermark detection.

Watermark embedding algorithm should be robust to such attacks. Watermark detection algorithm should decide whether watermark is present or absent in the video. If original video is used to make this decision, detector's efficiency is increased and this system is termed as non-blind or private or non-oblivious watermarking system. Non-Blind watermarking is very expensive in terms of storage and not a practical solution. Blind or public watermarking algorithms not require original video for watermark detection. These algorithms are more practical but detector's efficiency is low compared to one in private watermarking. In order to increase security of blind watermarking scheme it is desirable to use video dependent keys in the process of watermark generation.

In this paper we discussed various video watermarking generation techniques and their challenges and used it for Video authentication. Experimental results show that same watermark can be used for authentication as well as copyright protection.
\end{abstract}

\section{General Terms}

Digital Watermarking, Video watermarking

\section{Keywords}

Singular Value Decomposition, Attacks, DCT, LSB

\section{INTRODUCTION}

Video watermarking is one of the most popular techniques among the various watermarking techniques currently in use. This is because maximum occurrences of copyright violation and misuse happen for video media content [2]. Watermark generation, Watermark embedding and watermark extraction are three main modules of any watermarking process. Watermark can be broadly classified into two types robust and fragile. Robust watermark is generally used for copyright protection and fragile watermarks are mainly used for authentication and integrity verification.

In basic implementation, a watermark consist of sequence of real numbers $X=x 1, \ldots \ldots . . x n$. In practice value of watermark $\mathrm{xi}$ is chosen independently according to $\mathrm{N}(0,1)$ where $\mathrm{N}$ is a normal distribution. Sometimes Gaussian noise is also used as watermark. Logo image, audio clip or identifying information can be used as watermark.

In this paper we provide a survey of the latest techniques that are employed to watermark generation. The paper is organized in the following sections. In Section 2 we describe survey carried out. In Section 3 watermark generation module. In Section 4 we discuss the results. We conclude this paper in section 5 .

\section{WATERMARK GENERATION - A SURVEY}

Ingemar J. Cox et.al. [1], presented a secure (tamper resistant) methodology for copyright protection using spread spectrum technique. It can be extended for video watermarking but the problem is it needs original data while extracting watermark. Pik Wah Chan et.al. [1], proposed the novel algorithm, hybrid digital video watermarking, based on scene change technology and error correction code. They verified system resistant against attacks based on video characteristics and image processing techniques. They suggested enhancing the system by combining with audio watermarks for error correction capabilities and the hybrid scheme for attack resisting.

Yujie Zhang,et.al.[2] explained the new platform for professional video copyright protection. They used DCT, DWT and Neural Networks. They tested the platform for various copyright issues related to video, designed for MPEG Standard. They also concluded that at present only watermarking is not a complete solution for copyright protection, it needs other technical cooperation.

B.Shrinivas, et.al.[3] used audio fingerprints, Mel-Frequency Cepstral Coefficients(MFCC) and Vector Quantization (VQ) for automated internet movie piracy detection. Deepa Kundur, et.al.[4] presented robust logo watermarking using multi-resolution image fusion principles. This approach is called FuseMark. Dipti Mukherjee, et.al.[5] explained spatial domain digital watermarking of multimedia objects for buyer authentication. They used watermark in the form of a bit pattern specific for an individual buyer.

Chuhong Fei, et.al.[6] discussed analysis and design of authentication watermarking. Dimitrios Simitopoulos,et.al.[7] explained fast watermarking of MPEG-1/2 streams using compressed-domain perceptual embedding and a generalized correlator detector. They performed embedding and extraction without fully demultiplexing video, it leads to fast implementation. Mayank Vatsa, et.al.[8] used feature based 
RDWT watermarking for multimodal biometric system. They first computing embedding capacity and location using edge and corner phase congruency method and embedding and extracting voice data using redundant DWT.

Xinpeng Zhang, et.al.[9] used reference sharing mechanism for watermark self-embedding. This is fragile method for multimedia content authentication. Watermark used is a representation of host image contents. Chin-Chen Chang, et.al.[10] presented digital watermarking scheme using Human Visual Effects as a featured of images. They also used voting approach to improve correctness of extracted watermark. Chin-Yung Lin et.al[11], generated robust digital signature for image/video authentication. This signature generation is based on feature extraction and feature encryption. Kunvar Kalpesh N.,et.al.[12] described real time application to generate the differential time lapse video with pixel to pixel bit mapping algorithm. This is lossless visual watermarking scheme based on pixel difference value (PDV).

Lei Yang,Qian Chen, et.al.[13] proposed robust track-andtrace video watermarking. This algorithm is non-blind which required original video for watermark extraction. Vlado Kitanovski,et.al.[14] described watermark generation using image-dependent key for Image Authentication. Quantized Index Modulation of DCT coefficients are used for watermark embedding process. Andrew Tirkel, et.al.[15] presented a unique watermark generated for every image. This algorithm is in spatial domain and non-blind. Ron Schyndel,et.al.[16] described spread spectrum digital watermarking concepts and higher dimentional array construction and its implementation for watermarking. Samual Blake, et.al.[17] shown a construction for periodic ZCZ sequences using strong mathematical proofs. V.A.Mitekin, et.al[18] proposed new algorithm for collision-free watermark sequence generation.

\section{PROSPOSED SYSTEM}

Most of the video watermarking schemes are based on the techniques of image watermarking and directly applied to raw video or compressed video [1-3]. However, current image watermarking schemes are not capable of adequately protecting video data. Video watermarking introduces some issues which is not present in image watermarking. Due to large amounts of data and inherent redundancy between frames, video signals are highly susceptible to pirate attacks, including frame averaging, frame dropping, frame swapping, statistical analysis, etc. Applying a fixed image watermark to each frame in the video leads to problems of maintaining statistical and perceptual invisibility. Furthermore, such an approach is necessarily video independent; as the watermark is fixed while the frame changes. Applying independent watermarks to each frame also presents a problem. Regions in each video frame with little or no motion remain the same frame after frame. Motionless regions may be statistically compared or averaged to remove independent watermarks. In addition, video watermarking schemes must not use the original video during watermark detection as the video usually is in very large size [4][8] and it is inconvenient to store it twice. Following figure shows main modules in video watermarking process.

Algorithm 1 - Watermark Generation

$$
\begin{array}{ll}
\text { i. } & \text { Select Key frames(Fi) from input video } \\
\text { ii. } & \text { Divide frame into blocks } \\
\text { iii. } & \begin{array}{l}
\text { Calculate difference between blocks DC Values as } \\
\text { follows }
\end{array} \\
\text { Si }= & \sum_{j=1}^{8}(D C i-D C j) \\
\mathrm{Hi}=\left\{\begin{array}{l}
1 \text { if } S i \geq 0 \\
0 \text { if } S i<0
\end{array}\right.
\end{array}
$$

Where, $\mathrm{Hi}=$ one bit of frame signature derived from $\mathrm{k}^{\text {th }}$ block

i. Signature $\mathrm{H}$ is mapped to $32 \mathrm{X} 32$ Matrix

ii. Time Stamp and Copyright Information ( User Name and Organization) accepted as Date and String respectively and converted to binary values of matrix $16 \times 16$

iii. Combining iv and $\mathrm{v}$ we get generated matrix of size $64 \mathrm{X} 64$ which is used as watermark
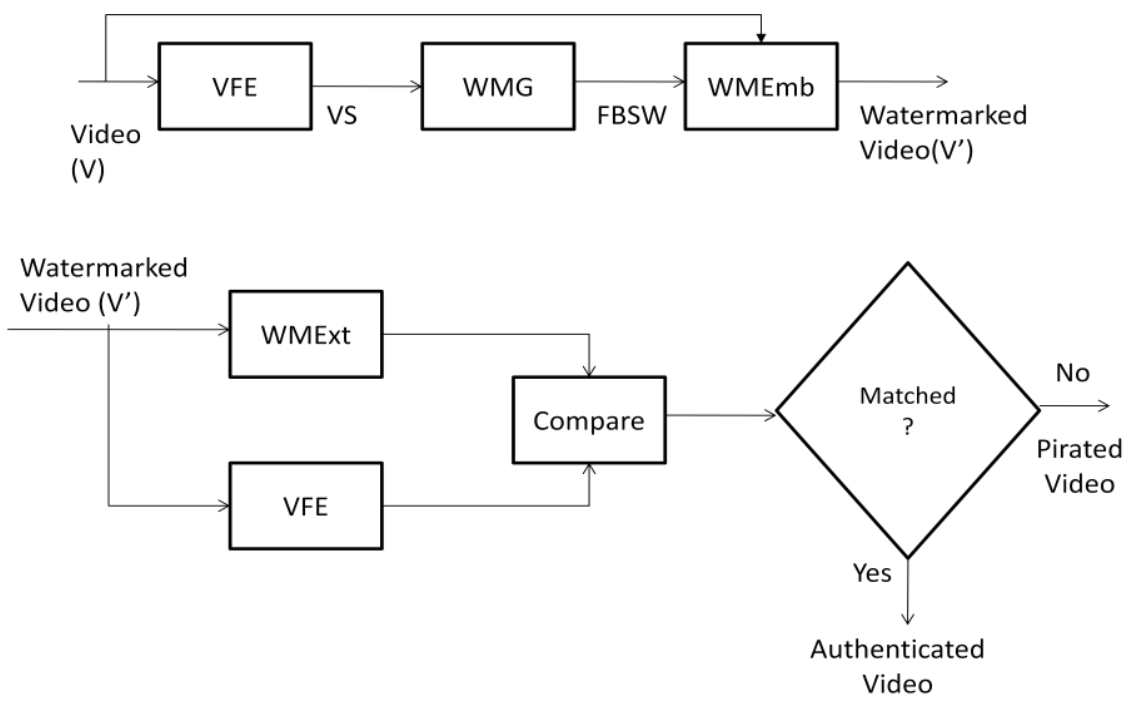

Fig. 1. Watermarking Scheme 
Algorithm 2 - Watermark Embedding

i. $\quad$ Select Frame(F) from Input Video (V)

ii. Split Frame (F) into 8X8 Blocks Bi and Each Block three matrices(U,S,V') using Singular Value Decomposition (SVD).

$\mathrm{B}=\mathrm{U} * \mathrm{~S} * \mathrm{~V}$,

iii. Scramble Final Bit Stream Watermark(FBSW) using Arnold Transform (W)

$\mathrm{x}^{\prime}=(\mathrm{x}+\mathrm{y}) \bmod \mathrm{N}$

$y^{\prime}=(x+2 y) \bmod N$

iv. Embedd Watermark into the frame as follow

$\mathrm{Z}=\bmod (\mathrm{S}(1,1), \mathrm{Q})$

if $(\mathrm{W}(\mathrm{i})==1)$

$$
\begin{aligned}
& \text { if }(\mathrm{Z}<=\mathrm{Q} / 4) \\
& \mathrm{S}(1,1)=\mathrm{S}(1,1)-\mathrm{Z}-\mathrm{Q} / 4
\end{aligned}
$$

else

$$
\mathrm{S}(1,1)=\mathrm{S}(1,1)-\mathrm{Z}+3 * \mathrm{Q} / 4
$$

end

else

$$
\begin{aligned}
& \text { if }(Z>=3 * Q / 4) \\
& S(1,1)=S(1,1)-Z+5 * Q / 4 \\
& \text { else } \\
& S(1,1)=S(1,1)-Z+Q / 4 \\
& \text { end }
\end{aligned}
$$

end

v. Embedded Block Bnew $=\mathrm{U}^{*}$ Snew $*$ V'

vi. Repeat till all embedded all watermark bits.

Algorithm 3 - Watermark Extraction

i. Select Frame(Fm) from Output Video (Vm)

ii. Split Frame (Fm) into 8X8 Blocks Bm and Each Block three matrices(Um,Sm,Vm') using Singular Value Decomposition (SVD).

iii. Extract Watermark into the frame as follow

$\mathrm{Z}=\bmod (\mathrm{S}(1,1), \mathrm{Q})$

if $(\mathrm{Z}>\mathrm{Q} / 2)$

$$
\mathrm{We}(\mathrm{i})=1 \text {; }
$$

else

$$
\mathrm{We}(\mathrm{i})=0 \text {; }
$$

end

iv. Repeat till all watermark bits extracted.

v. Apply reverse Arnodl's transform to unscramble the watermark

$\mathrm{x}=\left(2 \mathrm{x}^{\prime}-\mathrm{y}^{\prime}\right) \bmod \mathrm{N}$

\begin{tabular}{|c|c|c|c|c|}
\hline $\begin{array}{l}\text { Sr. } \\
\text { No }\end{array}$ & $\begin{array}{c}\text { Video } \\
\text { Sequence }\end{array}$ & Size & $\begin{array}{l}\text { No. of } \\
\text { Frames }\end{array}$ & Frame Size \\
\hline 1 & Atrium.avi & (22 MB) & $\begin{array}{l}431 \\
\text { Frames }\end{array}$ & $(640 \times 360)$ \\
\hline 2 & $\begin{array}{l}\text { Ghatatkoch. } \\
\text { mp4 }\end{array}$ & $\begin{array}{l}(1.62 \\
\mathrm{MB})\end{array}$ & $\begin{array}{l}299 \\
\text { Frames }\end{array}$ & (384X288) \\
\hline 3 & Akiyo.mp4 & $\begin{array}{l}(266 \\
\mathrm{KB})\end{array}$ & $\begin{array}{l}98 \\
\text { Frames }\end{array}$ & $(352 X 264)$ \\
\hline 4 & Kubako.mp4 & $\begin{array}{l}(2.65 \\
\mathrm{MB})\end{array}$ & $\begin{array}{l}302 \\
\text { Frames }\end{array}$ & $(480 \times 380)$ \\
\hline 5 & Lotr.mp4 & $\begin{array}{l}(596 \\
\mathrm{KB})\end{array}$ & $\begin{array}{l}301 \\
\text { Frames }\end{array}$ & (200X134) \\
\hline 6 & $\begin{array}{l}\text { Mb- } \\
\text { MBBS.mp4 }\end{array}$ & $\begin{array}{l}(3.36 \\
\mathrm{MB})\end{array}$ & $\begin{array}{l}299 \\
\text { Frames }\end{array}$ & $(600 \times 480)$ \\
\hline 7 & RN21.mp4 & $(1.5 \mathrm{MB})$ & $\begin{array}{l}299 \\
\text { Frames }\end{array}$ & $(480 \times 360)$ \\
\hline 8 & $\begin{array}{l}\text { SALSong.m } \\
\text { p4 }\end{array}$ & $\begin{array}{l}(3.31 \\
\mathrm{MB})\end{array}$ & $\begin{array}{l}302 \\
\text { Frames }\end{array}$ & $(884 X 480)$ \\
\hline 9 & Spring.mp4 & $\begin{array}{l}(9.35 \\
\mathrm{MB})\end{array}$ & $\begin{array}{l}299 \\
\text { Frames }\end{array}$ & $(1280 \times 720)$ \\
\hline 10 & Baby.mp4 & $\begin{array}{l}(4.41 \\
\mathrm{MB})\end{array}$ & $\begin{array}{l}1254 \\
\text { Frames }\end{array}$ & $(320 X 240)$ \\
\hline
\end{tabular}

$y=\left(-x^{\prime}+y^{\prime}\right) \bmod N$ vi. Extract copyright information from extracted watermark

vii. Compare it with original watermark

\section{RESULTS AND DISCUSSION}

Following table1 shows sample dataset used for experiments with varying sizes of frames and number of frames.

Table 1: Sample Video Data Set

Following table 2 shows result of watermark generation module- Copyright information accepted from user and timestamp is automatically accepted from the system.

Table 2: Watermark Generation Results

\begin{tabular}{|c|c|c|c|}
\hline \multicolumn{2}{|c|}{ Copyright Information } & \multirow{2}{*}{$\begin{array}{c}\text { Video } \\
\text { Sequence }\end{array}$} & $\begin{array}{c}\text { Generated } \\
\text { Watermark }\end{array}$ \\
\cline { 1 - 2 } Name & Address & & \\
\hline
\end{tabular}




\begin{tabular}{|l|l|l|l|l|}
\hline Chhaya & CCOEW & Baby.mp4 & & \\
\hline Archana & DYPIET & Atrium.avi & \\
\hline Govind & Pravara & GT.mp4 & \\
\hline Manoj & SKNCOE & Akiyo.mp4 & \\
\hline Baisa & Amrutvahini & MB.mp4 & \\
\hline & & \\
\hline
\end{tabular}

Following table 3 shows result of information extraction from extracted watermark

To evaluate performance and test robustness with respect to various attacks VideoPad Video Editor V 3.81 is used. To test robustness with respect to audio Audacity v 1.2.4 is used. Most of the implementation is done with JAVA jdk1.7 using Netbeans IDE v 7.0.1 and Xuggler 5.4. Some of the testing is done with MATLAB R2013.

Extracted watermark is compared with original watermark using Normalized Cross Correlation (NK)

$\mathrm{NK}=\frac{\sum_{i=1}^{M} \sum_{j=1}^{N}(x(i, j) \times y(i, j))}{\sum_{i=1}^{M} \sum_{j=1}^{N}(x(i, j))^{2}}$

Following table shows results with respect to various attacks

\begin{tabular}{|c|c|c|c|}
\hline \multirow[t]{2}{*}{ Video Files } & \multicolumn{3}{|l|}{ NK } \\
\hline & LSB & DCT & SVD \\
\hline Akiyo.mp4 & 0.99 & 1 & 1 \\
\hline Atrium.avi & 1 & 0.98 & 1 \\
\hline Baby.mp4 & 0.99 & 1 & 0.99 \\
\hline GT.mp4 & 0.98 & 1 & 1 \\
\hline MB.mp4 & 0.99 & 0.99 & 1 \\
\hline
\end{tabular}

Table 5 NK of Proposed Methods with frame dropping (10\%)

\begin{tabular}{|l|l|l|l|}
\hline \multirow{2}{*}{ Video Files } & \multicolumn{2}{|l|}{ NK } \\
\cline { 2 - 4 } & LSB & DCT & SVD \\
\hline Akiyo.mp4 & 0.23 & 0.81 & 0.89 \\
\hline Atrium.avi & 0.54 & 0.87 & 0.91 \\
\hline Baby.mp4 & 0.55 & 0.78 & 0.90 \\
\hline GT.mp4 & 0.43 & 0.79 & 0.88 \\
\hline MB.mp4 & 0.31 & 0.85 & 0.91 \\
\hline
\end{tabular}

Table 6 NK of Proposed Methods with frame swapping (10\%)

\begin{tabular}{|l|l|l|l|}
\hline \multirow{2}{*}{ Video Files } & \multicolumn{2}{|l|}{ NK } \\
\cline { 2 - 4 } & LSB & DCT & SVD \\
\hline Akiyo.mp4 & 0.33 & 0.89 & 0.92 \\
\hline Atrium.avi & 0.46 & 0.79 & 0.91 \\
\hline Baby.mp4 & 0.65 & 0.77 & 0.93 \\
\hline GT.mp4 & 0.48 & 0.86 & 0.9 \\
\hline MB.mp4 & 0.45 & 0.78 & 0.92 \\
\hline
\end{tabular}

Table 4 NK of Proposed Methods without Attacks

Table 3: Extracted Copyright information and video signature

\begin{tabular}{|c|c|c|c|c|c|c|c|c|c|}
\hline \multirow{2}{*}{$\begin{array}{c}\text { Extracted } \\
\text { Watermark }\end{array}$} & \multicolumn{2}{|c|}{ Copyright Information } & \multicolumn{6}{|c|}{ Time } & \multirow{2}{*}{$\begin{array}{c}\text { DC image } \\
\text { of Video }\end{array}$} \\
\hline & Name & Address & $Y r$ & $M n$ & $D t$ & $\mathrm{Hr}$ & $M i$ & Se & \\
\hline Akiyo.bmp & Manoj & SKNCOE & 2014 & 12 & 5 & 12 & 52 & 21 & \\
\hline Atrium.bmp & Archana & DYPIET & 2014 & 12 & 5 & 12 & 45 & 43 & \\
\hline Baby.bmp & Rakhi & CCOEW & 2014 & 12 & 5 & 12 & 28 & 43 & \\
\hline GT.bmp & Govind & Pravara & 2014 & 12 & 5 & 12 & 50 & 5 & \\
\hline MB_MBBS.bmp & Baisa & Amrutvahini & 2014 & 12 & 5 & 12 & 54 & 28 & \\
\hline
\end{tabular}




\section{CONCLUSION}

In this paper we proposed new watermarking scheme for video authentication and copyright protection. It is an attempt to summarize various watermarking generation techniques used for digital video. Experimental results show that our scheme is robust against attacks such as frame dropping, frame averaging and format changing. In future Visual cryptography can be used to secure watermark. HVS can also be used for block selection while embedding for more robustness against transcoding

\section{ACKNOWLEDGMENTS}

I am thankful to MKSSS's Cummins College of Engineering for Women, Padmashree Dr. D. Y. Patil College of Engineering and Technology and Sinhgad Institute of Technology and Science for encouragement and support

\section{REFERENCES}

[1] Ingemar J. Cox, Joe Kilian, F. Thomson Leighton, and Talal Shamoon, "Secure Spread Spectrum Watermarking for Multimedia," IEEE Transactions on Image Processing, Vol. 6, No. 12, pp. 1673-1687, December 1997.

[2] Pik Wah Chan, Michael R. Lyu, and Roland T. Chin, "A Novel Scheme for Hybrid Digital Video Watermarking: Approach, Evaluation and Experimentation," IEEE Transactions On Circuits and Systems for Video Technology, Vol. 15, No. 12, pp. 1638-1649, December 2005

[3] Yujie Zhang, Yuanyuan Zhang, "Research on Video Copyright Protection System," IEEE Consumer Electronics, Communications and Networks (CECNet), Proceedings of Second International Conference, pp. 1277 - 1281, 21-23 April 2012.

[4] B.Shrinivas, K. Venkara Rao, P. Suresh Verma, "Movie Piracy Detection Based on Audio Features using MelFrequency Cepstral Coefficients and Vector Quantization," International Journal of Soft Computing and Engineering, Vol. 02, No.4, pp. 27-31, September 2012.

[5] Deepa Kundur and Dimitrios Hatzinakos, "Toward Robust Logo Watermarking Using Multiresolution Image Fusion Principles," IEEE Transactions On Multimedia, Vol. 6, No. 1, pp.185-198, February 2004.

[6] Dipti Mukherjee, Subhamoy Maitra, and Scott Acton, "Spatial Domain Digital Watermarking of Multimedia Objects for Buyer Authentication," IEEE Transactions On Multimedia, Vol. 6, No. 1, pp. 1-15, 2004.

[7] Chuhong Fei, Deepa Kundur. and Raymond Kwong, "Analysis and Design of Authentication Watermarking," IEEE Transaction on Multimedia , Vol. 13, No. 2, pp. 126-144, 2004.
[8] Dimitrios Simitopoulos, Sotirios A. Tsaftaris, Nikolaos V. Boulgouris, Alexia Briassouli, Michael G. Strintzis, "Fast Watermarking of MPEG-1/2 Streams Using Compressed-Domain Perceptual Embedding and a Generalized Correlator Detector," EURASIP Journal on Applied Signal Processing, Vol. 8, pp. 1088-1106, July 2004.

[9] Mayank Vatsa, Richa Singh, Afzel Noore, "Feature based RDWT watermarking for multimodal biometric system," Science Direct Image and Vision Computing 27, 293-304, 2009

[10] Xinpeng Zhang, Shuozhong Wang, Zhenxing Qian, and Guorui Feng, "Reference Sharing Mechanism for Watermark Self-Embedding," IEEE Transactions On Image Processing, Vol. 20, No. 2,pp. 485-495, February 2011.

[11] Chin-Chen Chang, Kuo-Feng Hwang, "Digital Watermarking Scheme using Human Visual Effects," Informatica 24, pp. 505-511, 2000

[12] Chin-Yung Lin, Shih-Fu Chang, "Generating Robust Digital Signature for Image/Video Authentication," Multimedia and Security Workshop at ACM Multimedia, Briston, U.K., September 1998.

[13] Kunvar Kalpesh N., S.A.Patil, "Real time Application to Generate the Differential Time Lapse Video with Pixel to Pixel Bit Mapping Algorithm,"World Journal of Science and Technology, Vol 2, Issue 4, pp.123-126, 2012

[14] Lei Yang,Qian Chen, Jun Tian, Dapeng Wu, "Robust Track-and-Trace Video Watermarking,"

[15] Vlado Kitanovski, Dimitar Taskovski, "Watermark Generation using Image-dependent Key for Image Authentication," EUROCON,Serbia \& Montenegro, Blegrade, Nov. 2005.

[16] Andrew Tirkel, Thomas Hall, "A Unique Watermark for Every Image," IEEE Multimedia Transactions, Vol. 8, No. 4, pp. 30-37, Oct. 2001

[17] Ron Schyndel, Andrew Tirkel, Imants Svalbe, Thomas Hall, Charles Osborne, "Spread Spectrum Digital Watermarking Concepts and Higher Dimentional Array Construction," First International Online Symposium on Electronics Engineering, July 2000.

[18] Samual Blake, Andrew Tarkel, "A Construction for Periodic ZCZ Sequences,” Dec 2012

[19] V.A.Mitekin, E.I.Timbe, "A New Watermark Sequence Generation Algorithm for Collision-free Digital Watermarking," Eighth International Conference on Intelligent Information Hiding and Multimedia Signal Processing, 2012 